\title{
Diagnosing chronic thromboembolic pulmonary hypertension: current perspectives
}

\author{
This article was published in the following Dove Press journal: \\ Journal of Vascular Diagnostics \\ 4 September 2014 \\ Number of times this article has been viewed
}

\section{Charaka Hadinnapola \\ Deepa Gopalan \\ David $\mathrm{P}$ Jenkins}

Papworth Hospital National Health Service Foundation Trust, Papworth Everard, Cambridge, United Kingdom
Correspondence: David P Jenkins Department of Cardiothoracic Surgery, Papworth Hospital, Papworth Everard, Cambridge, CB23 3RE, United Kingdom Tel +44 I4 80364807

Fax +44 I4 80364709

Email david.jenkins@papworth.nhs.uk
Abstract: Chronic thromboembolic pulmonary hypertension is a rare and relatively poorly understood disease. It remains underdiagnosed and is often not recognized in primary and secondary care, as its symptoms are nonspecific and there are few clinical signs until late in the disease process. However, pulmonary endarterectomy (PEA) offers a potential cure for patients with this type of pulmonary hypertension; therefore, it is important that they are identified and diagnosed in a timely manner. PEA is associated with a $2.2 \%-5 \%$ risk of significant morbidity and mortality, even in experienced PEA centers. Therefore, once chronic thromboembolic pulmonary hypertension is diagnosed, further assessment of operability and patient selection is crucial. Assessment of operability involves determining the distribution and burden of chronic thromboembolic disease, assessing pulmonary hemodynamics, and assessing the functional impairment of the patient. Ventilation perfusion scintigraphy is of value in screening for the presence of chronic thromboembolic disease. However, computer tomography pulmonary angiography and magnetic resonance pulmonary angiography are now increasingly used to image the vascular occlusions directly. This allows assessment of the surgically accessible disease burden. Some centers still advocate conventional selective pulmonary angiography for the latter. Right-heart catheterization remains the gold standard for assessing pulmonary hemodynamics. Higher pulmonary vascular resistances are associated with poorer outcomes as well as increased risks at the time of surgery. This is in part because of the presence of more distal chronic thromboembolic material and distal pulmonary artery remodeling. However, in experienced centers, these patients are being operated on safely and with good outcomes. Patients with chronic thromboembolic disease, who have normal pulmonary hemodynamics, require careful functional assessments to determine the benefits of surgery.

Keywords: CTEPH, pulmonary endarterectomy, diagnosis

\section{Introduction}

Chronic thromboembolic pulmonary hypertension (CTEPH) is a rare disease with a relatively poorly understood pathogenesis. This hinders identifying those with and those at risk for the disease. Recent studies suggest that CTEPH is not as uncommon a complication after acute pulmonary embolism (PE) as once thought. ${ }^{1-3}$ However, the incidence of CTEPH in international registries is much less than would be expected from studies reporting the development of CTEPH in patients with acute PE. ${ }^{4}$ This suggests that CTEPH remains underdiagnosed in many. However, there is currently no evidence to suggest a universal screening program for CTEPH after an acute PE would be beneficial. ${ }^{3,5}$ The incidence of CTEPH remains too low to be considered an effective target for a screening program. It is important to highlight that these studies 
include all patients after acute PE. Patients who remain symptomatic after a period of adequate anticoagulation (usually around 3 months) should be considered for further investigation. In addition, a small but significant proportion (25\%) of patients diagnosed with CTEPH have no previous history of acute PE. ${ }^{4}$ Therefore, such screening programs will not detect these cases.

Unlike other forms of pulmonary arterial hypertension (PAH), in which treatment options remain limited, pulmonary endarterectomy (PEA) offers a potential cure for patients with CTEPH. ${ }^{6,7}$ The aim of PEA is the complete removal of organized thrombus from the pulmonary arterial circulation. This is a complex operation performed on cardiopulmonary bypass with deep hypothermic circulatory arrest. ${ }^{8,9}$ Careful patient selection is required even in experienced PEA centers, as the surgery is associated with a $2.2 \%-5 \%$ risk of significant morbidity and mortality. ${ }^{10,11}$ Timely diagnosis of CTEPH is crucial, as delays can lead to further development of distal vasculopathy and right heart dysfunction. After surgery, the right ventricle (RV) is able to reverse remodel, even in patients with significantly impaired RV function preoperatively. ${ }^{12}$

Understanding the pathogenesis of CTEPH helps explain the diagnostic and therapeutic difficulties facing clinicians. It also helps rationalize the investigative algorithms used in the assessment of CTEPH. In this review, we aim to briefly outline the current understanding of CTEPH pathogenesis before reviewing different aspects of our diagnostic algorithm. Diagnosis and operability assessment require assessment of pulmonary vascular hemodynamics, the distribution and burden of chronic thromboembolic material, and the functional effect on the patient.

\section{Pathogenesis}

CTEPH is thought to arise from a failure to resolve acute thromboembolic material within the pulmonary arteries. Numerous factors, including inflammation, failure of thrombolysis, endothelial dysfunction, and prothrombotic states have been associated with the development of CTEPH. ${ }^{4,13,14}$ Studies have reported an incidence of CTEPH after a first acute PE of between $0.5 \%$ and $10 \% .{ }^{14}$ However, the diagnosis is often delayed, especially in circumstances where an obvious precipitating acute embolic event has not been identified.

Histological descriptions of surgical specimens removed during PEA suggest that over time, there is replacement of acute fibrinous clot with fibrous organized tissue. ${ }^{15}$ This organized tissue adheres to the pulmonary arterial endothelial surface, forming web-like lesions and stenoses in the pulmonary arteries. This, in turn, disrupts the laminar pulsatile flow of blood in the pulmonary circulation. Under normal circumstances, the pulmonary circulation is a low-pressure, high-flow circuit. The vascular obstructions (caused by the chronic thromboembolic material) cause a progressive increase in mean pulmonary artery pressure (mPAP) and pulmonary vascular resistance (PVR). The RV is initially able to cope with this increase in afterload by increasing contractility and heart rate. To maintain cardiac output, the RV adapts by becoming hypertrophied. ${ }^{16}$

Over time, for reasons that are not completely understood, the adaptive remodeling of the RV fails. ${ }^{16}$ This results in progressive RV dilation and a reduction in cardiac output. This is associated with increasing exercise limitation and clinical features of right heart failure. Right heart failure is the most common cause of death in PAH. ${ }^{17,18}$

The increase in RV afterload, caused by macroscopic pulmonary vascular obstruction, is further exacerbated by changes to the distal pulmonary microcirculation. ${ }^{19-22}$ In this two-compartment model, it is hypothesized that there is remodeling of the pulmonary microcirculation in the perfused lung secondary to relative overperfusion and the subsequent increased pressure. Histologically, this remodeling is similar to the changes seen in idiopathic PAH (IPAH). ${ }^{21}$ This is responsible for further increases in MPAP and PVR. In patients with so-called "distal CTEPH," there is a combination of occluding chronic thromboembolic material, distributed mainly in subsegmental (or more distal) vessels, and remodeling of the microcirculation. As a consequence, PEA is often an unsuitable treatment option for this group, as the chronic thromboembolic material is not surgically accessible and the microcirculation changes remain postsurgery.

\section{Imaging}

Assessment of the distribution and burden of chronic thromboembolic material is essential for the diagnosis of CTEPH and for determining operability. During PEA, only chronic thromboembolic material in the more "proximal" pulmonary artery branches (up to a subsegmental level) is accessible. Therefore, it is critical to assess the burden of disease accessible by surgery preoperatively. It is also important to judge whether the hemodynamic compromise is in proportion to the vessel occlusion seen on imaging. The latter, often subjective, assessment is an indirect estimate of the contribution of the microvascular changes to the PVR. The decision to offer PEA to patients is dependent on the balance between the expected reductions in PVR postoperatively and the risks of surgery. This 
expected reduction in PVR correlates to the burden of proximal (surgically accessible) disease seen on imaging.

\section{Timing of assessments}

The timing of radiological assessments is crucial to minimizing the exposure of patients to unnecessary electromagnetic radiation and invasive procedures, especially in the context of acute PE, as the majority of patients will not develop CTEPH. Follow-up of patients after acute PE is heterogeneous. Patients are usually not routinely reviewed until at least 3 months after diagnosis and commencement of anticoagulation. It is assumed that within 3 months of anticoagulation, most acute thrombi should have dissipated. Therefore, persistent symptoms after 3 months of anticoagulation should be investigated further. Nijkeuter et al performed a systematic review of the resolution of thromboembolic disease after acute PE. ${ }^{23}$ They identified just four prospective studies that met their inclusion and exclusion criteria (25 studies were excluded). They report that more than $50 \%$ of patients have persistent radiographic changes based on ventilation perfusion scintigraphy (V/Q) or computer tomography pulmonary angiography (CTPA) evaluation at 6 months. They found that further improvements in radiological appearances were limited after this period. However, considerable variation is seen between studies. In a recent, large, retrospective study assessing resolution of acute PE with CTPAs, Aghayev et al demonstrated 94\% of patients showed complete resolution after just 90 days. ${ }^{24}$ However, this study is retrospective, and information regarding how the acute event was treated was not provided.

It is important to recognize that a proportion of patients often have evidence of chronic thromboembolic disease on follow-up imaging with no apparent hemodynamic or symptomatic effects. ${ }^{25}$ The natural history of such chronic disease is currently unknown. In our center, we currently advise that patients with persistent symptoms after acute PE should have follow-up investigations performed after a minimum of 3 months of anticoagulation.

\section{Ventilation perfusion scintigraphy}

The choice of imaging modality is equally important. V/Q scanning has a higher sensitivity for detection of perfusion defects compared with CTPA. ${ }^{26}$ In a retrospective study by Tunariu et al, ${ }^{26} \mathrm{~V} / \mathrm{Q}$ scans had a sensitivity of around $97 \%$ compared with $51 \%$ for CTPAs for detecting chronic thromboembolic material. However, CTPAs had a higher specificity compared with V/Q scans ( $99 \%$ versus $92.5 \%$, respectively). Therefore, V/Q scans are useful as a screening test to exclude the presence of chronic thromboembolic material in the pulmonary circulation, especially when investigating the cause of proven PAH. In the prospective international CTEPH registry, $99.4 \%$ of patients had evidence of perfusion abnormalities with V/Q scans. ${ }^{27}$

There is also a potential role for $\mathrm{V} / \mathrm{Q}$ scans in inferring the presence of distal thromboembolic disease that may otherwise not be seen directly on CTPA, magnetic resonance pulmonary angiography (MRPA), or selective pulmonary angiography (Figure 1). This is particularly useful when the disease burden directly imaged is less than would be expected for the measured hemodynamic profile. This mismatch can arise from remodeling of distal pulmonary arteries or distal occlusions that are not imaged directly by CTPA. ${ }^{28}$ However, when determining suitability for PEA, assessment of the "proximal" disease burden and distribution is crucial. As a consequence, direct imaging of these occlusions is required.

\section{Computer tomography pulmonary angiography and magnetic resonance pulmonary angiography}

At this time, largely based on consensus opinion, selective pulmonary angiography with digital subtraction angiography (DSA) is considered the gold standard for assessing operability by determining disease distribution and burden. ${ }^{529-31}$ Some studies have previously suggested that CTPA and MRPA are adequate in identifying pulmonary vascular occlusions compared with DSA, ${ }^{32,33}$ whereas other studies have reported on the superiority of CTPA in delineating central thrombotic material over DSA, as well as in identifying partial occlusions. A recent direct comparison between electrocardiogram-gated CTPA, contrast-enhanced MRPA, and DSA by Ley et al suggests CTPA was superior to both MRPA and DSA at all levels of the pulmonary vasculature. ${ }^{34}$ Electrocardiogram-gated CTPA provided 100\% sensitivity

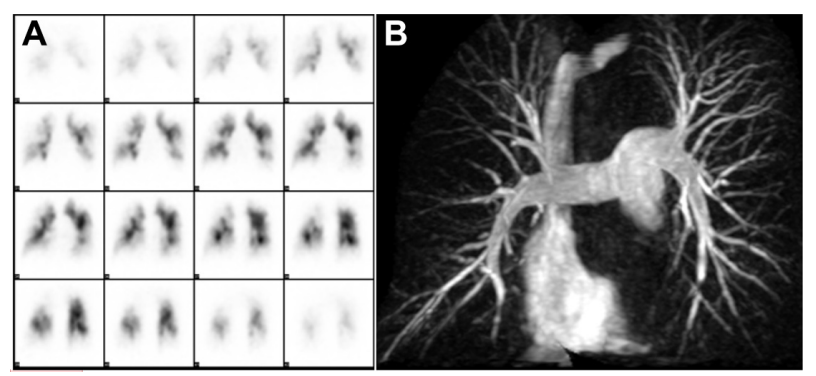

Figure I (A) Coronal single-photon emission computed tomography perfusion scintigram showing large bilateral perfusion defects. (B) Corresponding magnetic resonance pulmonary angiogram shows mixed disease with a predominately distal component. 
and specificity for detecting occlusive thrombi at the level of the main or lobar pulmonary arteries. This compares with a sensitivity of $83.1 \%$ and specificity of $98.6 \%$ with MRPA, and a sensitivity of $65.7 \%$ and specificity of $100 \%$ with DSA at the same level. At segmental levels, CTPA had a sensitivity of $100 \%$ and specificity of $99 \%$. This compares with a sensitivity of $87.7 \%$ and specificity of $98.1 \%$ with MRPA and a sensitivity of $75.8 \%$ and specificity of $100 \%$ with DSA.

CTPA has the added advantage of assessing the lung parenchyma for coexisting respiratory pathology. This can be of importance in assessing the surgical risks associated with PEA. Additional useful radiological features seen on CTPA include mosaic perfusion and simple measurements of right ventricular size and structure. Dual-energy CT angiography (Figure 2) has been shown to provide additional information regarding the pulmonary microvasculature that is not obtained by traditional CTPA. ${ }^{35,36}$ It provides an assessment of pulmonary blood volume at a specific point in time, rather than being a true measure of lung perfusion. However, there is a strong correlation between dual-energy-derived lung perfusion and mosaic attenuation. Although Dournes et al found moderate agreement between V/Q and dual-energy perfusion imaging, the former should still be considered the gold standard tool for screening. ${ }^{36}$ Dual-energy CT angiography also improves vascular enhancement, allowing assessment of bronchial artery collaterals, which are often dilated and are of prognostic significance in CTEPH. ${ }^{37}$

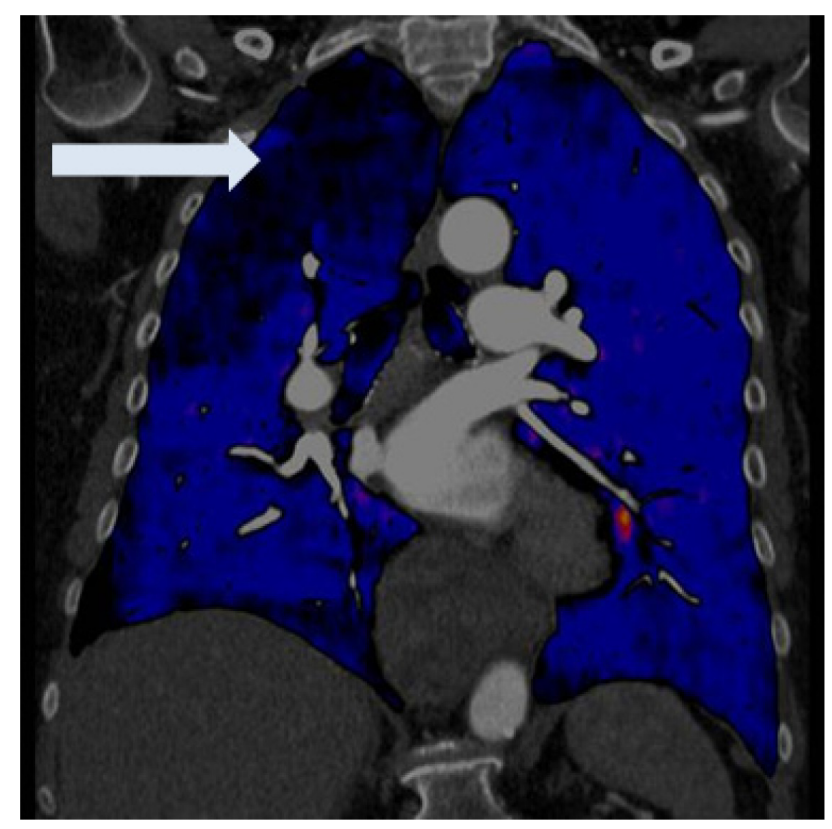

Figure 2 Coronal view of a dual-energy computed tomography pulmonary angiogram demonstrating large segmental perfusion defects in the right lung (arrow) in a patient with thromboembolic disease.
Magnetic resonance imaging is a fast-evolving field (Figure 3). In addition to assessing the pulmonary vasculature, it has the additional advantage of providing information regarding RV structure and function, as well as estimated cardiac output from aortic and pulmonary artery flow calculations. ${ }^{38,39}$ Lung perfusion magnetic resonance imaging protocols have a high sensitivity for diagnosing CTEPH and have better spatial resolution compared with V/Q. ${ }^{40}$ However, apart from in specialist centers with experience with magnetic resonance imaging perfusion protocols, its place in the diagnostic algorithm requires further investigation.

Pulmonary artery sarcoma is a rare differential diagnosis that needs to be considered in patients with presumed CTEPH. Findings on CTPA that may suggest this diagnosis include the complete obliteration of the vessel lumen, but with relative enlargement of the vessel diameter, as well as a convex margin of the occlusive material and the presence of occlusive material in the pulmonary trunk at the level of the valve leaflets. ${ }^{41}$ Positron emission tomography may show characteristic increased uptake of 18-fluoro-deoxyglucose within a pulmonary sarcoma, but the situation can be confused if the sarcoma includes associated in situ thrombotic material. ${ }^{42}$

\section{Other modalities}

Balloon pulmonary angioplasty is a novel potential therapeutic intervention for CTEPH. Although its use has been highlighted in several case series, it is yet to be proven as a treatment modality for this disease. ${ }^{43-46}$ Many of these studies make use of additional imaging modalities such as optical coherence tomography and intravascular ultrasound to select lesions suitable for angioplasty. ${ }^{43,46-48}$ In the largest case series, involving 68 patients and reported by Mizoguchi et al,

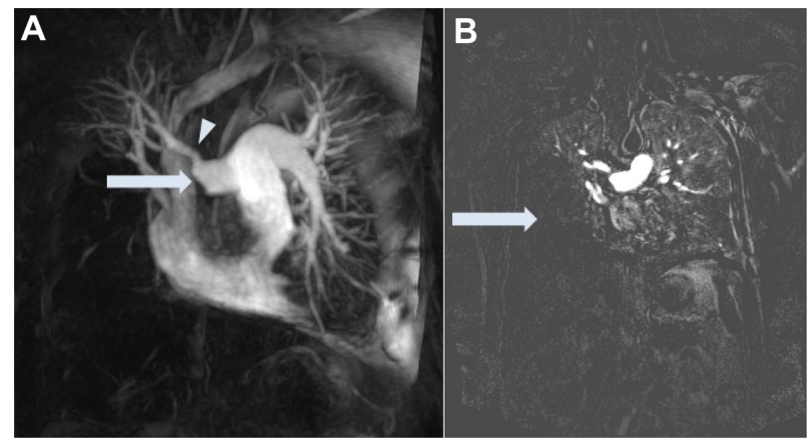

Figure 3 (A) High spatial resolution magnetic resonance pulmonary angiogram showing proximal amputation of right-sided pulmonary arteries (arrow) distal to the origin of the apical segment of the upper lobe (arrow head). There is predominately distal disease in the left lower lobe and lingula. (B) Corresponding magnetic resonance perfusion image shows a large perfusion defect in the right middle and lower lobes (arrow) and mid and lower lobes of the left lung. 
intravascular ultrasound was used to determine the size of balloon required for angioplasty. ${ }^{43}$ The role of these modalities will become clearer as more experience and consensus regarding balloon pulmonary angioplasty are reached.

At our center, current practice is to use VQ scans as a screening test for CTEPH and in assessing distal disease burden. Before PEA, two imaging modalities are used to determine distribution and burden of disease. Both patient factors (such as comorbidities, claustrophobia, and metal implants) and technical factors will guide this selection. In our experience, CTPA and MRPA together provide sufficient information to make safe and accurate decisions regarding suitability for surgery.

\section{Echocardiography}

Transthoracic echocardiography provides a simple bedside assessment on cardiac structure and function. Because of the right ventricular shape and location, detailed assessment is harder with echocardiography compared with the left ventricle. However, experienced operators are able to provide detailed descriptions of cardiac function that correlate to invasive hemodynamic measures. ${ }^{49} \mathrm{RV}$ parameters on echocardiography, especially tricuspid annular plane systolic excursion, correlate to cardiac magnetic resonance imaging (CMR) derived parameters of RV function. ${ }^{50}$ The use of real-time three-dimensional echocardiography also improves assessment of the RV. ${ }^{51}$

Echocardiography has been evaluated as a screening tool for CTEPH, as it is noninvasive and has no extra radiation exposure. Although the value of screening for CTEPH in all patients after acute PE remains unverified, as discussed earlier, there is certainly value in using it initially in the assessment of patients who remain symptomatic. After this, more invasive tests, such as right heart catheterization and imaging with CTPA, can be used to confirm the diagnosis. In addition, it can be used for longitudinal follow-up of RV size and function after PEA. ${ }^{12,52}$

\section{Right heart catheterization}

Right heart catheterization remains the best way of accurately determining pulmonary hemodynamics and right ventricular function and is essential for the diagnosis of pulmonary hypertension. Cardiac magnetic resonance imaging is another tool that provides information regarding right heart function. Although pulmonary artery pressures cannot be measured directly with cardiac magnetic resonance imaging, several parameters have been found to correlate with this. By definition, CTEPH requires the presence of PAH.
Current guidelines define this as an mPAP of $25 \mathrm{mmHg}$ or higher and a pulmonary capillary wedge pressure (PCWP) of $15 \mathrm{mmHg}$ or lower when measured at rest by right heart catheterization. ${ }^{30,53}$

The presence of vascular occlusions in CTEPH can make assessment of PCWP difficult, as they prevent "wedging" of the Swan-Ganz catheter into a distal arteriole. Assessment of PCWP is important in determining the presence of left heart disease. This is possible with coexisting coronary artery disease. At our center, all patients older than 50 years or with significant risk factors predisposing them to coronary artery disease undergo coronary angiography (with a view to performing coronary artery bypass grafting at the time of PEA). If there are concerns regarding elevated PCWP, left heart catheterization allows assessment of the left ventricular end diastolic pressure. Cardiac magnetic resonance imaging and echocardiography are also useful tools in assessing left ventricular function.

High PVR has been associated with poor outcomes after PEA. This is likely a reflection of increased distal disease burden and small vessel vasculopathy. A PVR higher than 1,000 dyn $\times$ second $\times \mathrm{cm}^{-5}$ correlates with increased postoperative mortality $(4.1 \%$ versus $1.6 \%) .{ }^{11}$ While in the prospective international CTEPH registry reported by Mayer et al, a PVR higher than 1,200 dyn $\times$ second $\times \mathrm{cm}^{-5}$ is associated with a failure to normalize pulmonary hemodynamics, as well as higher mortality, postoperatively. ${ }^{27}$ It must be stressed that in experienced centers, operating on patients with such high PVRs has been shown to be safe and effective. ${ }^{54}$ Higher PVRs should not preclude PEA, as these patients have potentially the most to gain from surgery. There are hemodynamic and functional benefits from a reduction in disease burden, even if the pulmonary hemodynamics are not completely normalized postoperatively. ${ }^{55}$ The development and approval of novel therapeutic drugs for patients with residual pulmonary hypertension after PEA is likely to further improve outcome in this group. ${ }^{56}$

In addition to PVR, pulmonary artery compliance is increasingly recognized as an important predictor in outcome in both IPAH and CTEPH (especially postoperative measurements)..$^{57-59}$ de Perrot et al examined changes in PVR and compliance before and after PEA. They showed that postoperative compliance was the only factor on multivariate analysis to predict functional improvement to New York Heart Association class $1 .{ }^{60}$ Ghio et al have also recently shown that postoperative compliance is a good predictor of exercise capacity at 3 months after PEA. ${ }^{58}$ 
Unfortunately, attempts at predicting postoperative outcomes based on baseline hemodynamic parameters have been unsuccessful. The product of PVR and compliance (called RC time) is a reflection of the pressure decay in the pulmonary artery in diastole. MacKenzie Ross et al found that the RC times of patients with proximal disease who had normalization of their mPAPs after PEA were significantly lower than those who had more distal disease and did not normalize their hemodynamics postoperatively. ${ }^{61}$ Unfortunately, as there was considerable overlap between the two groups, this relatively simple test cannot be used to predict postoperative outcomes. Similar difficulties have limited the use of pulmonary artery occlusion pressure analysis to predict outcomes. ${ }^{62,63}$ At present, there are no risk-stratification tools for identifying patients who are less likely to normalize their pulmonary hemodynamics postoperatively.

On the other extreme of the hemodynamic spectrum are patients with chronic thromboembolic material in their pulmonary arteries, but who do not meet the hemodynamic criteria for PAH (this will be referred to as chronic thromboembolic disease, or CTED). ${ }^{30}$ However, many of these patients still have exercise limitations. Several mechanisms may cause this exercise limitation. For example, Kovacs et al performed a systematic review of hemodynamic measurements in healthy volunteers at rest and exercise.$^{64}$ From this data, the mPAP in healthy individuals at rest in a supine position is $13.95 \pm 3.3 \mathrm{mmHg}$. A proportion of patients with CTED will fall into a grey area between $20 \mathrm{mmHg}$ (ie, two standard deviations above the mean reported pressure in health) and $25 \mathrm{mmHg}$. Therefore, these patients' hemodynamics should not necessarily be considered normal, even if they do not meet the hemodynamic definition of PAH.

In addition, McCabe et al demonstrated that conductance catheter-derived pressure volume loop morphology and tau (a measure of right ventricular diastolic relaxation) is significantly different in patients with CTED (mPAP, $17 \pm 5 \mathrm{mmHg}$ ) compared with controls (patients undergoing patent foramen ovale closure; mPAP, $15 \pm 3 \mathrm{mmHg}$ ). ${ }^{65}$ This suggests there are early changes in right ventricular function occurring in patients with CTED.

In health, although mPAP rises slightly with exercise, this is associated with a reduction in PVR because of the increase in cardiac output and recruitment of additional pulmonary vascular beds. Bonderman et al demonstrated that in 13 patients with persistent dyspnea after PEA (but with a resting $\mathrm{mPAP} \leq 25 \mathrm{mmHg}$ ), exercise resulted in an increase in PVR and a drop in pulmonary artery compliance. ${ }^{59}$ The opposite was seen in the control population. Biomechanical changes to the pulmonary artery as a result of PEA need to be considered as a possible explanation for these hemodynamic findings. However, it would be interesting to assess whether similar changes are seen in CTED patients preoperatively. Assessments of exercise hemodynamics are not currently part of PAH guidelines because of insufficient evidence, but they may provide useful additional information. ${ }^{53}$

Finally, increased physiological dead space and ventilatory inefficiency have been demonstrated in patients with CTEPH compared with those with IPAH. ${ }^{66}$ This is likely caused by the vascular obstructions in CTEPH. van der Plas et al have shown that physiological dead space correlates with patient-reported dyspnea in CTEPH. ${ }^{67}$ Furthermore, after PEA, they demonstrated a decrease in physiological dead space that correlated with resting Borg scores. In CTED patients, McCabe et al have demonstrated that ventilatory efficiency (as measured with the ventilatory equivalent ratio for carbon dioxide $\left[\mathrm{Ve} / \mathrm{VCO}_{2}\right]$ at the anaerobic threshold and the $\mathrm{Ve} / \mathrm{VCO}_{2}$ slope) is reduced compared with controls. ${ }^{68}$ Therefore, these changes in ventilatory efficiency may provide another explanation for exercise limitation in this group of patients with CTED. PEA may result in an improvement in dead space, thereby improving ventilatory efficiency, dyspnea, and exercise capacity.

At this time, several centers are offering PEA surgery to patients with minimal hemodynamic compromise at rest, but who demonstrate functional and physiological limitations. ${ }^{11,69,70}$ Very little is known about outcome of PEA in this group of patients.

\section{Functional assessments}

The 6 minute walk test provides a relatively simple measure of exercise capacity. It has been validated and used extensively in the assessment of CTEPH. ${ }^{55,71}$ However, it remains a submaximal test and is effort-dependent. It is also less useful in the elderly, who may be limited by musculoskeletal problems that may reduce the distance walked. Cardiopulmonary exercise testing, as described earlier, overcomes many of the limitations of the 6 minute walk test and provides more physiological correlates. Therefore, it provides an excellent research and clinical tool to investigate the functional effects of therapeutic interventions.

\section{Conclusion}

The Fifth World Symposium on Pulmonary Hypertension, held in 2013, recognized the importance of suspecting CTEPH in those at risk for or with symptoms, confirming the diagnosis and determining the suitability for and risks of PEA. ${ }^{31}$ The patient pathway should include these three 
steps to prevent diagnostic delay and initiate appropriate management plans. This requires the diagnostic assessments described earlier being evaluated together by an expert multidisciplinary CTEPH team. This is especially true for higher-risk patients, such as those with PVRs higher than 1,200 dyn $\times$ second $\times \mathrm{cm}^{-5}$, where centers with more experience are more likely to offer surgery and more likely to have better outcomes. Several centers have reported on the learning curve for PEA. ${ }^{10,11,70}$ More-experienced centers report lower mortality rates, despite treating more high-risk cases. This learning curve also applies to the multidisciplinary team of surgeons, radiologists, and pulmonary hypertension physicians, who all bring their own expertise to the decisionmaking process.

Without doubt, PEA remains the treatment of choice in patients with surgically accessible disease and no significant medical comorbidities that would preclude surgery. However, with the licensing of riociguat for the treatment of inoperable and residual CTEPH after PEA, and the emergence of balloon pulmonary angioplasty, other treatment modalities should be considered for those with predominately "distal" CTEPH or with significant medical comorbidities. ${ }^{43,56}$ With increasing therapeutic choices for patients with CTEPH, careful assessment will become even more essential to ensure the most appropriate treatment modality is offered.

In this review, we summarize the most important diagnostic tools used to detect CTEPH and also explain how operability is assessed.

\section{Disclosure}

The authors report no conflicts of interest in this work.

\section{References}

1. Pengo V, Lensing AW, Prins MH, et al; Thromboembolic Pulmonary Hypertension Study Group. Incidence of chronic thromboembolic pulmonary hypertension after pulmonary embolism. $N$ Engl $J$ Med. 2004;350(22):2257-2264

2. Lang IM. Chronic thromboembolic pulmonary hypertension - not so rare after all. $N$ Engl J Med. 2004;350(22):2236-2238.

3. Klok FA, van Kralingen KW, van Dijk AP, Heyning FH, Vliegen HW, Huisman MV. Prospective cardiopulmonary screening program to detect chronic thromboembolic pulmonary hypertension in patients after acute pulmonary embolism. Haematologica. 2010;95(6):970-975.

4. Pepke-Zaba J, Delcroix M, Lang I, et al. Chronic thromboembolic pulmonary hypertension $(\mathrm{CTEPH})$ : results from an international prospective registry. Circulation. 2011;124(18):1973-1981.

5. Mehta S, Helmersen D, Provencher S, et al; Canadian Thoracic Society Pulmonary Vascular Disease - CTEPH CPG Development Committee; Canadian Thoracic Society Canadian Respiratory Guidelines Committee. Diagnostic evaluation and management of chronic thromboembolic pulmonary hypertension: a clinical practice guideline. Can Respir J. 2010;17(6):301-334.
6. Jenkins DP, Madani M, Mayer E, et al. Surgical treatment of chronic thromboembolic pulmonary hypertension. Eur Respir J. 2013;41(3): 735-742.

7. Galiè N, Corris PA, Frost A, et al. Updated treatment algorithm of pulmonary arterial hypertension. J Am Coll Cardiol. 2013;62(25) (Suppl):D60-D72.

8. Jamieson SW, Kapelanski DP. Pulmonary endarterectomy. Curr Probl Surg. 2000;37(3):165-252.

9. Vuylsteke A, Sharples L, Charman G, et al. Circulatory arrest versus cerebral perfusion during pulmonary endarterectomy surgery (PEACOG): a randomised controlled trial. Lancet. 2011;378(9800):1379-1387.

10. Freed DH, Thomson BM, Tsui SS, et al. Functional and haemodynamic outcome 1 year after pulmonary thromboendarterectomy. Eur $J$ Cardiothorac Surg. 2008;34(3):525-529, discussion 529-530.

11. Madani MM, Auger WR, Pretorius V, et al. Pulmonary endarterectomy: recent changes in a single institution's experience of more than 2,700 patients. Ann Thorac Surg. 2012;94(1):97-103, discussion 103.

12. Dittrich HC, Nicod PH, Chow LC, Chappuis FP, Moser KM, Peterson KL. Early changes of right heart geometry after pulmonary thromboendarterectomy. J Am Coll Cardiol. 1988;11(5):937-943.

13. Bonderman D, Jakowitsch J, Adlbrecht C, et al. Medical conditions increasing the risk of chronic thromboembolic pulmonary hypertension. Thromb Haemost. 2005;93(3):512-516.

14. Lang IM, Pesavento R, Bonderman D, Yuan JX. Risk factors and basic mechanisms of chronic thromboembolic pulmonary hypertension: a current understanding. Eur Respir J. 2013;41(2):462-468.

15. Bernard J, Yi ES. Pulmonary thromboendarterectomy: a clinicopathologic study of 200 consecutive pulmonary thromboendarterectomy cases in one institution. Hum Pathol. 2007;38(6):871-877.

16. Bogaard HJ, Abe K, Vonk Noordegraaf A, Voelkel NF. The right ventricle under pressure: cellular and molecular mechanisms of right-heart failure in pulmonary hypertension. Chest. 2009;135(3): 794-804.

17. Sandoval J, Bauerle O, Palomar A, et al. Survival in primary pulmonary hypertension. Validation of a prognostic equation. Circulation. 1994;89(4):1733-1744.

18. Riedel M, Stanek V, Widimsky J, Prerovsky I. Longterm follow-up of patients with pulmonary thromboembolism. Late prognosis and evolution of hemodynamic and respiratory data. Chest. 1982;81(2):151-158.

19. Moser KM, Bloor CM. Pulmonary vascular lesions occurring in patients with chronic major vessel thromboembolic pulmonary hypertension. Chest. 1993;103(3):685-692.

20. Galiè N, Kim NH. Pulmonary microvascular disease in chronic thromboembolic pulmonary hypertension. Proc Am Thorac Soc. 2006;3(7):571-576.

21. Yi ES, Kim H, Ahn H, et al. Distribution of obstructive intimal lesions and their cellular phenotypes in chronic pulmonary hypertension. A morphometric and immunohistochemical study. Am J Respir Crit Care Med. 2000;162(4 Pt 1):1577-1586.

22. Moser KM, Braunwald NS. Successful surgical intervention in severe chronic thromboembolic pulmonary hypertension. Chest. 1973;64(1):29-35.

23. Nijkeuter M, Hovens MM, Davidson BL, Huisman MV. Resolution of thromboemboli in patients with acute pulmonary embolism: a systematic review. Chest. 2006;129(1):192-197.

24. Aghayev A, Furlan A, Patil A, et al. The rate of resolution of clot burden measured by pulmonary CT angiography in patients with acute pulmonary embolism. AJR Am J Roentgenol. 2013;200(4):791-797.

25. Sanchez O, Helley D, Couchon S, et al. Perfusion defects after pulmonary embolism: risk factors and clinical significance. J Thromb Haemost. 2010;8(6):1248-1255.

26. Tunariu N, Gibbs SJ, Win Z, et al. Ventilation-perfusion scintigraphy is more sensitive than multidetector CTPA in detecting chronic thromboembolic pulmonary disease as a treatable cause of pulmonary hypertension. J Nucl Med. 2007;48(5):680-684. 
27. Mayer E, Jenkins D, Lindner J, et al. Surgical management and outcome of patients with chronic thromboembolic pulmonary hypertension: results from an international prospective registry. J Thorac Cardiovasc Surg. 2011;141(3):702-710.

28. Azarian R, Wartski M, Collignon MA, et al. Lung perfusion scans and hemodynamics in acute and chronic pulmonary embolism. J Nucl Med. 1997;38(6):980-983.

29. Coulden R. State-of-the-art imaging techniques in chronic thromboembolic pulmonary hypertension. Proc Am Thorac Soc. 2006;3(7):577-583.

30. Galiè N, Hoeper MM, Humbert M, et al; Task Force for Diagnosis and Treatment of Pulmonary Hypertension of European Society of Cardiology (ESC); European Respiratory Society (ERS); International Society of Heart and Lung Transplantation (ISHLT). Guidelines for the diagnosis and treatment of pulmonary hypertension. Eur Respir J. 2009;34(6):1219-1263.

31. Kim NH, Delcroix M, Jenkins DP, et al. Chronic thromboembolic pulmonary hypertension. J Am Coll Cardiol. 2013;62(25)(Suppl): D92-D99.

32. Reichelt A, Hoeper MM, Galanski M, Keberle M. Chronic thromboembolic pulmonary hypertension: evaluation with 64-detector row CT versus digital substraction angiography. Eur J Radiol. 2009;71(1):49-54.

33. Ley S, Kauczor HU, Heussel CP, et al. Value of contrast-enhanced MR angiography and helical CT angiography in chronic thromboembolic pulmonary hypertension. Eur Radiol. 2003;13(10):2365-2371.

34. Ley S, Ley-Zaporozhan J, Pitton MB, et al. Diagnostic performance of state-of-the-art imaging techniques for morphological assessment of vascular abnormalities in patients with chronic thromboembolic pulmonary hypertension (CTEPH). Eur Radiol. 2012;22(3):607-616.

35. Hoey ET, Mirsadraee S, Pepke-Zaba J, Jenkins DP, Gopalan D, Screaton NJ. Dual-energy CT angiography for assessment of regional pulmonary perfusion in patients with chronic thromboembolic pulmonary hypertension: initial experience. AJR Am J Roentgenol. 2011;196(3):524-532.

36. Dournes G, Verdier D, Montaudon M, et al. Dual-energy CT perfusion and angiography in chronic thromboembolic pulmonary hypertension: diagnostic accuracy and concordance with radionuclide scintigraphy. Eur Radiol. 2014;24(1):42-51.

37. Kauczor HU, Schwickert HC, Mayer E, Schweden F, Schild HH, Thelen M. Spiral CT of bronchial arteries in chronic thromboembolism. J Comput Assist Tomogr. 1994;18(6):855-861.

38. Swift AJ, Rajaram S, Condliffe R, et al. Diagnostic accuracy of cardiovascular magnetic resonance imaging of right ventricular morphology and function in the assessment of suspected pulmonary hypertension results from the ASPIRE registry. J Cardiovasc Magn Reson. 2012;14(1):40.

39. Swift AJ, Rajaram S, Hurdman J, et al. Noninvasive estimation of PA pressure, flow, and resistance with CMR imaging: derivation and prospective validation study from the ASPIRE registry. JACC Cardiovasc Imaging. 2013;6(10):1036-1047.

40. Rajaram S, Swift AJ, Telfer A, et al. 3D contrast-enhanced lung perfusion MRI is an effective screening tool for chronic thromboembolic pulmonary hypertension: results from the ASPIRE Registry. Thorax. 2013;68(7):677-678.

41. Attinà $\mathrm{D}$, Niro $\mathrm{F}$, Tchouanté $\mathrm{P}$, et al. Pulmonary artery intimal sarcoma. Problems in the differential diagnosis. Radiol Med (Torino). 2013;118(8):1259-1268.

42. Rajaram S, Swift AJ, Davies C, et al. Primary pulmonary artery sarcoma and coexisting chronic thromboembolic pulmonary hypertension. Am J Respir Crit Care Med. 2013;188(5):e7-e8.

43. Mizoguchi H, Ogawa A, Munemasa M, Mikouchi H, Ito H, Matsubara H. Refined balloon pulmonary angioplasty for inoperable patients with chronic thromboembolic pulmonary hypertension. Circ Cardiovasc Interv. 2012;5(6):748-755.
44. Kataoka M, Inami T, Hayashida K, et al. Percutaneous transluminal pulmonary angioplasty for the treatment of chronic thromboembolic pulmonary hypertension. Circ Cardiovasc Interv. 2012;5(6):756-762.

45. Andreassen AK, Ragnarsson A, Gude E, Geiran O, Andersen R. Balloon pulmonary angioplasty in patients with inoperable chronic thromboembolic pulmonary hypertension. Heart. 2013;99(19):1415-1420.

46. Sugimura K, Fukumoto Y, Satoh K, et al. Percutaneous transluminal pulmonary angioplasty markedly improves pulmonary hemodynamics and long-term prognosis in patients with chronic thromboembolic pulmonary hypertension. Circ J. 2012;76(2):485-488.

47. Tatebe S, Fukumoto Y, Sugimura K, et al. Optical coherence tomography as a novel diagnostic tool for distal type chronic thromboembolic pulmonary hypertension. Circ J. 2010;74(8): $1742-1744$

48. Tatebe S, Fukumoto Y, Sugimura K, et al. Optical coherence tomography is superior to intravascular ultrasound for diagnosis of distal-type chronic thromboembolic pulmonary hypertension. Circ J. 2013;77(4):1081-1083.

49. Blanchard DG, MaloufPJ, Gurudevan SV, et al. Utility of right ventricularTei index in the noninvasive evaluation of chronic thromboembolic pulmonary hypertension before and after pulmonary thromboendarterectomy. JACC Cardiovasc Imaging. 2009;2(2):143-149.

50. Sato T, Tsujino I, Ohira H, et al. Validation study on the accuracy of echocardiographic measurements of right ventricular systolic function in pulmonary hypertension. J Am Soc Echocardiogr. 2012;25(3): 280-286.

51. Gopal AS, Chukwu EO, Iwuchukwu CJ, et al. Normal values of right ventricular size and function by real-time 3-dimensional echocardiography: comparison with cardiac magnetic resonance imaging. J Am Soc Echocardiogr. 2007;20(5):445-455.

52. D'Armini AM, Zanotti G, Ghio S, et al. Reverse right ventricular remodeling after pulmonary endarterectomy. J Thorac Cardiovasc Surg. 2007;133(1):162-168.

53. Hoeper MM, Bogaard HJ, Condliffe R, et al. Definitions and diagnosis of pulmonary hypertension. J Am Coll Cardiol. 2013;62(25) (Suppl):D42-D50.

54. Thistlethwaite PA, Kemp A, Du L, Madani MM, Jamieson SW. Outcomes of pulmonary endarterectomy for treatment of extreme thromboembolic pulmonary hypertension. J Thorac Cardiovasc Surg. 2006;131(2):307-313.

55. Condliffe R, Kiely DG, Gibbs JS, et al. Improved outcomes in medically and surgically treated chronic thromboembolic pulmonary hypertension. Am J Respir Crit Care Med. 2008;177(10): 1122-1127.

56. Ghofrani HA, D'Armini AM, Grimminger F, et al; CHEST-1 Study Group. Riociguat for the treatment of chronic thromboembolic pulmonary hypertension. $N$ Engl J Med. 2013;369(4):319-329.

57. Mahapatra S, Nishimura RA, Sorajja P, Cha S, McGoon MD. Relationship of pulmonary arterial capacitance and mortality in idiopathic pulmonary arterial hypertension. J Am Coll Cardiol. 2006;47(4): 799-803.

58. Ghio S, Morsolini M, Corsico A, et al. Pulmonary arterial compliance and exercise capacity after pulmonary endarterectomy. Eur Respir J. 2014;43(5):1403-1409.

59. Bonderman D, Martischnig AM, Vonbank K, et al. Right ventricular load at exercise is a cause of persistent exercise limitation in patients with normal resting pulmonary vascular resistance after pulmonary endarterectomy. Chest. 2011;139(1):122-127.

60. de Perrot M, McRae K, ShargallY, et al. Early postoperative pulmonary vascular compliance predicts outcome after pulmonary endarterectomy for chronic thromboembolic pulmonary hypertension. Chest. 2011;140(1):34-41.

61. MacKenzie Ross RV, Toshner MR, Soon E, Naeije R, Pepke-Zaba J. Decreased time constant of the pulmonary circulation in chronic thromboembolic pulmonary hypertension. Am J Physiol Heart Circ Physiol. 2013;305(2):H259-H264. 
62. Toshner M, Suntharalingam J, Fesler P, et al. Occlusion pressure analysis role in partitioning of pulmonary vascular resistance in CTEPH. Eur Respir J. 2012;40(3):612-617.

63. Kim NH, Fesler P, Channick RN, et al. Preoperative partitioning of pulmonary vascular resistance correlates with early outcome after thromboendarterectomy for chronic thromboembolic pulmonary hypertension. Circulation. 2004;109(1):18-22.

64. Kovacs G, Berghold A, Scheidl S, Olschewski H. Pulmonary arterial pressure during rest and exercise in healthy subjects: a systematic review. Eur Respir J. 2009;34(4):888-894.

65. McCabe C, White PA, Hoole SP, et al. Right ventricular dysfunction in chronic thromboembolic obstruction of the pulmonary artery: a pressure-volume study using the conductance catheter. J Appl Physiol (1985). 2014;116(4):355-363.

66. Zhai Z, Murphy K, Tighe H, et al. Differences in ventilatory inefficiency between pulmonary arterial hypertension and chronic thromboembolic pulmonary hypertension. Chest. 2011;140(5):1284-1291.
67. van der Plas MN, Reesink HJ, Roos CM, van Steenwijk RP, Kloek JJ, Bresser P. Pulmonary endarterectomy improves dyspnea by the relief of dead space ventilation. Ann Thorac Surg. 2010;89(2):347-352.

68. McCabe C, Deboeck G, Harvey I, et al. Inefficient exercise gas exchange identifies pulmonary hypertension in chronic thromboembolic obstruction following pulmonary embolism. Thromb Res. 2013;132(6): 659-665.

69. Klepetko W, Mayer E, Sandoval J, et al. Interventional and surgical modalities of treatment for pulmonary arterial hypertension. J Am Coll Cardiol. 2004;43(12)(Suppl S):73S-80S.

70. Mayer E. Surgical and post-operative treatment of chronic thromboembolic pulmonary hypertension. Eur Respir Rev. 2010; 19(115):64-67.

71. Suntharalingam J, Goldsmith K, Toshner M, et al. Role of NT-proBNP and 6MWD in chronic thromboembolic pulmonary hypertension. Respir Med. 2007;101(11):2254-2262.

\section{Publish your work in this journal}

Journal of Vascular Diagnostics is an international, peer-reviewed journal of diagnostics, focusing on non invasive vascular investigation methods involved in the evaluation of vascular diseases. The journal is committed to the rapid publication in the fields of vascular diseases Original research, review, case reports, expert opinion and commentaries

\section{Dovepress}

are all considered for publication. The manuscript management system is completely online and includes a very quick and fair peer-review system, which is all easy to use. Visit http://www.dovepress.com/testimonials.php to read real quotes from published authors.

Submit your manuscript here: http://www.dovepress.com/journal-of-vascular-diagnostics-journal 